\title{
Adaptação do teste de condutividade elétrica para sementes de Moringa oleifera
}

\author{
Maria Luiza de Souza Medeiros ${ }^{1 *}$, Guilherme Vinícius Gonçalves de Pádua ${ }^{1}$, Márcio Dias Pereira ${ }^{1}$ \\ ${ }^{1}$ Universidade Federal do Rio Grande do Norte, RN 160, Km 03, CEP 59280-000, Macaíba, RN, Brasil
}

"Autor correspondente:

luizamedeiros30@hotmail.com

Termos para indexação:

Moringaceae

Potencial fisiológico

Vigor

Index terms:

Moringacea

Physiologic potential

Vigor

Recebido em 15/11/2016

Aprovado em 08/09/2017

Publicado em 29/09/2017

doi: 10.4336/2017.pfb.37.91.1384

\begin{abstract}
Resumo - O objetivo desse trabalho foi adaptar e testar a eficiência da metodologia para teste de condutividade elétrica na avaliação da qualidade de sementes de Moringa oleifera Lam. Para a caracterização fisiológica das sementes, quatro lotes de sementes foram avaliados pelos testes de germinação, emergência de plântula, índice de velocidade de emergência, primeira contagem de emergência, comprimento e massa seca de plântulas e teste de frio. O teste de condutividade elétrica foi avaliado a $25^{\circ} \mathrm{C}$, em 4, 8, 12, 16 e 24 h de imersão em $75 \mathrm{~mL}$ e $125 \mathrm{~mL}$ de água destilada, utilizando-se 25 e 50 sementes. Empregou-se o delineamento inteiramente casualizado. Os melhores resultados foram obtidos quando utilizou-se 50 sementes imersas em $75 \mathrm{~mL}$ ou 125 $\mathrm{mL}$ de água destilada por $4 \mathrm{~h}$. O teste de condutividade elétrica adaptado para moringa mostrou-se eficiente em ranquear os lotes de sementes em diferentes níveis de vigor, podendo ser usado com eficiência na avaliação da sua qualidade fisiológica.
\end{abstract}

\section{Adaptation of electrical conductivity test for Moringa oleifera seeds}

\begin{abstract}
This study aimed to adapt and test the efficiency of electrical conductivity methodology test in quality evaluation of Moringa oleifera Lam seeds. For physiological characterization four seed sets were evaluated by tests of germination, seedlings emergency, speed of emergency index, emergency first count, seedlings length and dry mass and cold test. The electrical conductivity test was carried out at $25^{\circ} \mathrm{C}$ for $4,8,12,16$ and $24 \mathrm{~h}$ of immersion in 75 or $125 \mathrm{~mL}$ of distilled water using 25 or 50 seeds. A completely randomized design was used. The best results were obtained when using 50 seeds immersed in $75 \mathrm{~mL}$ or $125 \mathrm{~mL}$ of distilled water for $4 \mathrm{~h}$. The electrical conductivity test adapted to moringa seeds was efficient in ranking sets of different vigor levels. The test may be efficiently used for physiological quality evaluation of moringa seeds.
\end{abstract}

\section{Introdução}

Moringa oleifera Lam., pertencente à família das Moringaceae, é nativa da Índia e amplamente cultivada na Etiópia, Sudão e muitos países da Ásia e América Central (Karadi et al., 2006). Popularmente conhecida como moringa, é uma planta rústica, de rápido crescimento, resistente à seca e de alto valor nutricional no que diz respeito às vitaminas e minerais. Essas características fazem com que a espécie seja apontada como alternativa para o semiárido brasileiro e torna-se ainda mais atrativa por ser de fácil cultivo, baixo custo de produção e de alto rendimento (Ferreira et al., 2008).

Além de sua importância econômica por sua aplicação na indústria, pela busca por alternativas que aliviem a 
falta de alimentação humana e animal em períodos muito secos, como também suas características nutracêuticas, tem feito com que aumente a procura por sementes e mudas de moringa de alta qualidade.

Nesse contexto, a análise de sementes constitui-se como etapa fundamental para garantir a qualidade do material produzido. Por sua vez, seu uso é fator determinante para o êxito do empreendimento agroflorestal, permitindo, dessa forma, que conhecimentos concretos das condições de um lote de sementes sejam obtidos ao mesmo tempo que decisões corretas, no que se refere à escolha de sementes de boa qualidade, sejam tomadas (Valadares \& Paula, 2008; Tokuhisa et al., 2009).

Por esse motivo, é necessário avaliar o potencial fisiológico dos lotes de sementes através de testes de vigor, possibilitando a identificação de diferenças de qualidade dos mesmos, com resultados gerados em período de tempo relativamente curto, o que possibilita aos tecnologistas, produtores e pesquisadores a obtenção de um adequado estande sob distintas condições de campo.

Entre os testes de vigor utilizados atualmente, destaca-se o de condutividade elétrica, sendo a qualidade das sementes avaliada indiretamente por meio da determinação da quantidade de lixiviados na solução de embebição das sementes (Sponchiado, 2014). Esse teste pressupõe que o vigor da semente está diretamente relacionado com a integridade do sistema de membranas celulares. As sementes de baixo vigor tendem a apresentar desorganização na estrutura das membranas celulares, permitindo aumento na lixiviação de solutos (Marcos Filho, 2005). Portanto, sementes menos vigorosas (mais deterioradas) liberam maior quantidade de lixiviados, como consequência da menor velocidade de reestruturação das membranas (Vieira et al., 2002).

Diversos são os fatores que podem afetar os resultados no teste de condutividade elétrica, como espécie avaliada, tempo de imersão, teor de água, peso, volume, número de sementes, dosagem utilizada na imersão das sementes, integridade e idade das sementes, genótipo e temperatura (Rodrigues et al., 2006). Nesse sentido, há algumas indicações na literatura para a condução do teste de condutividade elétrica para sementes de espécies cultivadas, como a metodologia sugerida por Silva et al. (2014) para avaliação de sementes de feijão, utilizando 25 sementes por $4 \mathrm{~h}$ em $75 \mathrm{~mL}$ de água. Para milho recomenda-se a embebição das sementes tanto a 50 quanto a $75 \mathrm{~mL}$ de água, por $24 \mathrm{~h}$ ou $48 \mathrm{~h}$ (Ribeiro et al., 2009). No entanto, não há na literatura informações sobre a metodologia específica para condução desse teste para sementes de moringa.

Diante do exposto, o presente trabalho teve como objetivo adaptar a metodologia do teste de condutividade elétrica e analisar a sua eficiência na avaliação da qualidade de sementes de moringa.

\section{Material e métodos}

Foram utilizadas sementes de Moringa oleifera coletadas manualmente em sete árvores matrizes, provenientes de quatro localidades: Parelhas, RN (Lote $1-06^{\circ} 41^{\prime} 16^{\prime}$ 'S e 36 39'27'W), Ceará-mirim, RN (Lote 2 - 05 $38^{\circ} 04^{\prime \prime}$ S e $35^{\circ} 25^{\prime} 32^{\prime \prime} \mathrm{W}$ ), Caiçara, RN (Lote $3-05^{\circ} 04^{\prime} 48^{\prime \prime}$ S e $36^{\circ} 04^{\prime} 07^{\prime \prime} W$ ) e São Paulo, SP (Lote $4-23^{\circ} 32^{\prime} 56^{\prime \prime} \mathrm{S}$ e $\left.46^{\circ} 38^{\prime} 20^{\prime \prime} \mathrm{W}\right)$.

Cada lote era composto por 1.100 sementes. Essas foram caracterizadas fisiologicamente pelos testes e determinações apresentados a seguir.

$\mathrm{O}$ teor de água das sementes foi determinado pelo método da estufa a $105 \pm 3{ }^{\circ} \mathrm{C}$ por $24 \mathrm{~h}$, utilizando-se duas subamostras de cada lote, com aproximadamente $4,5 \mathrm{~g}$. Os resultados foram expressos em porcentagem de teor de água em base úmida (Brasil, 2009).

No teste de germinação, foram utilizados quatro subamostras de 50 sementes por lote, sendo utilizado o substrato rolo de papel (tipo Germitest ${ }^{\circledR}$ ). As sementes foram distribuídas sobre duas folhas de papel e cobertas com uma terceira, previamente umedecidas com água destilada, na quantidade equivalente a três vezes seu peso seco. Os rolos contendo as sementes foram acondicionados em sacos plásticos e mantidos em germinador do tipo Biochemical Oxygen Demand (BOD) a $30{ }^{\circ} \mathrm{C}$. As avaliações foram realizadas aos quatro e dez dias após a semeadura, sendo determinada a porcentagem de plântulas normais, de acordo com as recomendações das Regras para análise de sementes (Brasil, 2009).

O teste de emergência de plântulas foi conduzido em casa de vegetação, utilizando-se bandejas plásticas, com capacidade volumétrica aproximada de $9 \mathrm{~L}$, contendo areia previamente lavada, peneirada, esterilizada e umedecida inicialmente com $60 \%$ de sua capacidade de retenção máxima, conforme sugerido por Brasil (2009). Quatro subamostras de 25 sementes foram distribuídas nas bandejas, sendo irrigadas, sempre que necessário, para manter o substrato na capacidade de campo. $\mathrm{O}$ 
cálculo da quantidade de água adicionada foi efetuado pesando-se $500 \mathrm{~g}$ de areia colocada em um filtro de papel. Em seguida, foi adicionada uma quantidade de água previamente determinada. Decorridos aproximadamente 15 min, todo o excesso de água drenado foi utilizado para determinar o cálculo, por diferença, da quantidade de água que ficou retida na areia (100\%). A quantidade de água equivalente a $60 \%$ foi adicionada aos $500 \mathrm{~g}$ de areia (Brasil, 2009). Foram realizadas contagens diárias, do sétimo ao décimo quarto dia após a semeadura, registrando-se o número de plântulas emergidas, para a determinação da porcentagem de emergência. A primeira contagem de emergência consistiu do registro do número de plântulas normais (\%) obtidas aos sete dias após o início do teste de emergência.

O índice de velocidade de emergência (IVE) foi determinado mediante a contagem diária do número de plântulas normais identificadas no teste de emergência em campo, sendo calculado conforme a metodologia proposta por Maguire (1962).

O comprimento total e a massa seca de plântulas foram determinados após a contagem final do teste de emergência. As plântulas normais foram destorroadas, lavadas, secas com papel toalha e posteriormente submetidas a medições com o auxílio de uma régua graduada (resultados em cm). Após a medição, as plântulas foram acondicionadas em sacos de papel do tipo Kraft, sendo colocados em estufa regulada a $65^{\circ} \mathrm{C}$, até obtenção de peso constante (48 h). Decorrido esse período, as amostras foram pesadas em balança analítica com precisão de 0,001 g (resultados em g) (Nakagawa, 1999).

Para o teste de frio, foram utilizadas quatro repetições de 50 sementes para cada lote. Utilizou-se o mesmo procedimento descrito para o teste de germinação para a confecção dos rolos. No entanto, as sementes foram mantidas em câmara do tipo BOD por 10 dias, regulada a $15{ }^{\circ} \mathrm{C}$. Após este período, os rolos foram transferidos para germinador previamente regulado a $30{ }^{\circ} \mathrm{C}$, onde permaneceram por mais nove dias, quando foi realizada a contagem e determinado o número de plântulas normais (\%).

Para a adequação da metodologia do teste de condutividade elétrica, foram utilizados quatro lotes, cinco períodos de embebição $(4,8,12,16$ e 24 h), dois volumes de água destilada $(75 \mathrm{~mL}$ e $125 \mathrm{~mL}) \mathrm{e}$ duas quantidades de sementes por repetição (25 e 50). Foram utilizadas quatro repetições por lote e, antes da leitura, as sementes foram previamente pesadas em balança analítica com precisão de $0,001 \mathrm{~g}$ e colocadas em recipientes (copos) plásticos com capacidade para $400 \mathrm{~mL}$, cobertos com papel alumínio. Esses foram mantidos em germinador previamente regulado a $25^{\circ} \mathrm{C}$. Após cada período de embebição, a condutividade elétrica foi medida com o auxílio de um condutivímetro da marca Tecnal ${ }^{\circledR}$ modelo TEC-4MP. Antes do início das leituras foi realizada a leitura da prova em branco (somente da água destilada). Os resultados das leituras foram diminuídos dos valores da condutividade elétrica obtidos da prova em branco e então divididos pelos respectivos valores de massa das amostras das sementes (resultados expressos em $\mu \mathrm{S} \mathrm{cm}^{-1} \mathrm{~g}^{-1}$ de semente).

O delineamento estatístico utilizado foi o inteiramente casualizado. Os resultados foram submetidos à análise de variância, sendo as médias em cada tratamento comparadas pelo teste de Tukey $(\mathrm{p}<0,05)$.

\section{Resultados}

O teor de água das sementes foi semelhante entre os lotes estudados (Tabela 1), com variação de até 1,17\%.

Tabela 1. Valores de teor de água (TA), germinação (G), emergência $(E)$, primeira contagem de emergência (PCE), índice de velocidade de emergência (IVE), comprimento de plântulas em campo (CP), massa seca de plântulas em laboratório (MSL), massa seca de plântulas em campo (MSC) e teste de frio (TF) de sementes de Moringa oleifera Lam. de quatro lotes de sementes.

\begin{tabular}{|c|c|c|c|c|c|c|c|c|c|}
\hline Lotes & TA $(\%)$ & G (\%) & E (\%) & PCE $(\%)$ & IVE & CP $(\%)$ & MSL (\%) & MSC (\%) & $\mathrm{TF}(\%)$ \\
\hline 1 & 8,48 & $87,00 \mathrm{~b}$ & $44,00 \mathrm{c}$ & $11,00 \mathrm{~b}$ & $1,20 \mathrm{~b}$ & $12,70 \mathrm{ab}$ & $42,70 \mathrm{a}$ & $89,00 \mathrm{a}$ & $43,50 \mathrm{~b}$ \\
\hline 2 & 7,36 & $97,00 \mathrm{ab}$ & $84,00 \mathrm{ab}$ & $49,00 \mathrm{a}$ & $2,80 \mathrm{a}$ & $9,53 \mathrm{~b}$ & $31,60 \mathrm{~b}$ & $64,30 \mathrm{~b}$ & $93,00 \mathrm{a}$ \\
\hline 3 & 7,31 & $99,00 \mathrm{a}$ & $92,00 \mathrm{a}$ & $37,00 \mathrm{ab}$ & $2,70 \mathrm{a}$ & $16,00 \mathrm{a}$ & $28,40 \mathrm{~b}$ & $88,80 \mathrm{ab}$ & $95,00 \mathrm{a}$ \\
\hline 4 & 8,00 & $94,00 \mathrm{ab}$ & $69,00 \mathrm{~b}$ & $32,0 \mathrm{ab}$ & $2,10 \mathrm{a}$ & $16,50 \mathrm{a}$ & $38,00 \mathrm{a}$ & $112,70 \mathrm{a}$ & $91,00 \mathrm{a}$ \\
\hline
\end{tabular}

As médias seguidas pela mesma letra, na coluna, não diferem estatisticamente pelo teste de Tukey, ao nível de 5\% de probabilidade. 
Foi possível observar que apenas o lote 1 diferiu dos demais, apresentando o pior desempenho nos testes de germinação, emergência, primeira contagem de emergência, índice de velocidade de emergência e teste de frio (Tabela 1), sendo classificado como o de pior qualidade fisiológica.

No teste de emergência (Tabela 1), o lote 3 apresentou o melhor desempenho, superior também aos lotes 1 e 4 .

Observou-se que os lotes 1, 3 e 4 foram semelhantes para a variável comprimento de plântulas em campo (Tabela 1), apresentando melhor qualidade fisiológica que o lote 2.

Maior massa seca de plântulas em laboratório (Tabela 1), foi observada nos lotes 1 e 4 . Em condições de campo, o lote 2 apresentou o pior desempenho, não sendo observada diferença entre os demais.

O teste de condutividade elétrica não apresentou boa separação entre os lotes de sementes, considerando a qualidade fisiológica, quando utilizou-se volume de $75 \mathrm{~mL}, 25$ sementes, nos diferentes períodos de embebição testados (Tabela 2). Porém, quando se utilizou 50 sementes, independente do período de embebição, o teste de condutividade elétrica foi suficientemente sensível para separar os lotes em níveis de vigor. O lote 3 resultou em menor valor de condutividade, equivalendo ao de melhor potencial fisiológico, e o lote 4 o com pior desempenho neste teste.

Na Tabela 3, encontram-se os resultados do teste de condutividade elétrica, avaliando-se o volume de 125 $\mathrm{mL}$ em amostras de 25 e 50 sementes. Foi possível observar um comportamento da lixiviação de exsudatos semelhante ao verificado com o uso de $75 \mathrm{~mL}$. No entanto, com 50 sementes houve melhor estratificação dos lotes em diferentes níveis de vigor, com o período de embebição de 4 h, apontando o lote 3 como o mais vigoroso e o 4 o de menor vigor.

Tabela 2. Condutividade elétrica $\left(\mu \mathrm{S} \mathrm{cm}^{-1} \mathrm{~g}^{-1}\right)$ de quatro lotes de sementes de Moringa oleifera Lam. embebidas por $4,8,12,16$ e 24 h, em amostras de 25 e 50 sementes, acondicionadas em $75 \mathrm{~mL}$ de água destilada.

\begin{tabular}{|c|c|c|c|c|c|c|c|c|c|c|}
\hline \multirow{2}{*}{ Lotes } & \multicolumn{5}{|c|}{25 sementes } & \multicolumn{5}{|c|}{50 sementes } \\
\hline & $4 h$ & $8 \mathrm{~h}$ & $12 \mathrm{~h}$ & $16 \mathrm{~h}$ & $24 \mathrm{~h}$ & $4 h$ & $8 \mathrm{~h}$ & $12 \mathrm{~h}$ & $16 \mathrm{~h}$ & $24 \mathrm{~h}$ \\
\hline 1 & $58,30 \mathrm{a}$ & 67,33 a & $74,57 \mathrm{a}$ & $77,26 \mathrm{a}$ & $91,34 \mathrm{ab}$ & $68,60 \mathrm{~b}$ & $88,22 \mathrm{~b}$ & $101,68 \mathrm{~b}$ & $111,98 \mathrm{~b}$ & $141,78 \mathrm{~b}$ \\
\hline 2 & $27,20 \mathrm{~b}$ & $36,07 \mathrm{bc}$ & $41,84 \mathrm{~b}$ & $46,82 \mathrm{~b}$ & $64,21 \mathrm{bc}$ & $43,52 \mathrm{c}$ & $59,41 \mathrm{c}$ & $67,08 \mathrm{c}$ & $74,74 \mathrm{c}$ & $95,98 \mathrm{c}$ \\
\hline 3 & $19,62 \mathrm{~b}$ & $24,35 \mathrm{c}$ & $27,78 \mathrm{~b}$ & $30,38 \mathrm{~b}$ & $38,69 \mathrm{c}$ & $20,69 d$ & $28,74 \mathrm{~d}$ & $34,49 \mathrm{~d}$ & $39,23 \mathrm{~d}$ & $53,55 \mathrm{~d}$ \\
\hline 4 & $49,36 \mathrm{a}$ & $58,16 \mathrm{ab}$ & 68,00 a & $78,34 \mathrm{a}$ & $124,22 \mathrm{a}$ & $93,78 \mathrm{a}$ & $113,80 \mathrm{a}$ & $133,62 \mathrm{a}$ & $156,05 \mathrm{a}$ & $237,04 \mathrm{a}$ \\
\hline CV (\%) & 23,97 & 23,75 & 22,44 & 22,52 & 22,76 & 17,37 & 16,04 & 16,22 & 15,89 & 14,79 \\
\hline
\end{tabular}

As médias seguidas pela mesma letra, na coluna, não diferem estatisticamente pelo teste de Tukey, ao nível de 5\% de probabilidade.

Tabela 3. Condutividade elétrica $\left(\mu \mathrm{S} \mathrm{cm}^{-1} \mathrm{~g}^{-1}\right)$ de quatro lotes de sementes de Moringa oleifera Lam. embebidas por $4,8,12,16$ e $24 \mathrm{~h}$ em amostras de 25 e 50 sementes, acondicionadas em $125 \mathrm{~mL}$ de água destilada.

\begin{tabular}{|c|c|c|c|c|c|c|c|c|c|c|}
\hline \multirow{2}{*}{ Lotes } & \multicolumn{5}{|c|}{25 sementes } & \multicolumn{5}{|c|}{50 sementes } \\
\hline & $4 h$ & $8 \mathrm{~h}$ & 12 & 16 & 24 & $4 h$ & $8 h$ & $12 \mathrm{~h}$ & $16 \mathrm{~h}$ & $24 \mathrm{~h}$ \\
\hline 1 & $25,67 \mathrm{a}$ & $30,15 \mathrm{a}$ & 33,57 a & $35,53 \mathrm{a}$ & $45,17 \mathrm{~b}$ & $37,10 \mathrm{~b}$ & $48,19 \mathrm{~b}$ & $54,85 \mathrm{~b}$ & $60,80 \mathrm{~b}$ & $78,07 \mathrm{~b}$ \\
\hline 2 & $13,57 \mathrm{~b}$ & $19,55 \mathrm{~b}$ & $22,01 \mathrm{~b}$ & $25,30 \mathrm{~b}$ & $35,95 \mathrm{bc}$ & $23,93 \mathrm{c}$ & $34,72 \mathrm{~b}$ & $41,33 \mathrm{~b}$ & $47,85 \mathrm{~b}$ & $67,27 \mathrm{~b}$ \\
\hline 3 & $11,68 \mathrm{~b}$ & $14,26 \mathrm{~b}$ & $16,54 \mathrm{~b}$ & $18,28 \mathrm{~b}$ & $23,79 \mathrm{c}$ & $11,94 \mathrm{~d}$ & $16,86 \mathrm{c}$ & $19,43 \mathrm{c}$ & $22,44 \mathrm{c}$ & $30,46 \mathrm{c}$ \\
\hline 4 & $24,85 \mathrm{a}$ & $32,65 \mathrm{a}$ & $36,93 \mathrm{a}$ & $43,27 \mathrm{a}$ & $64,97 \mathrm{a}$ & $56,36 \mathrm{a}$ & $73,69 \mathrm{a}$ & $80,97 \mathrm{a}$ & $94,60 \mathrm{a}$ & $142,62 \mathrm{a}$ \\
\hline CV (\%) & 15,56 & 13,81 & 13,12 & 13,51 & 14,47 & 16,65 & 16,91 & 17,56 & 17,19 & 15,77 \\
\hline
\end{tabular}

As médias seguidas pela mesma letra, na coluna, não diferem estatisticamente pelo teste de Tukey, ao nível de $5 \%$ de probabilidade. 


\section{Discussão}

Um fator imprescindível para a obtenção de resultados consistentes na análise de sementes é a uniformidade do teor de água inicial, para padronização das avaliações (Krzyzanowski et al., 1991). Os resultados encontrados no presente trabalho (Tabela 1) encontram-se dentro da faixa tolerável, não comprometendo a comparação da qualidade fisiológica entre diferentes lotes de sementes (Marcos Filho, 2005).

Segundo Marcos Filho \& Novembre (2009) quando os resultados dos testes de germinação são muito diferentes, não há necessidade de testes de vigor, pois o próprio teste de germinação conseguiria detectar diferenças na qualidade fisiológica das sementes, por se tratar de um teste conduzido sob condições controladas de laboratório. No entanto, não foram observadas variações de germinação muito acentuadas (Tabela 1), sendo importante a condução de testes de vigor.

$\mathrm{O}$ teste de emergência é eficiente para avaliar o potencial fisiológico de lotes de sementes, por ser conduzido em condições que simulam aquelas que as sementes estarão sujeitas em campo. Através desse teste pode-se determinar o índice de velocidade de emergência, que objetiva detectar diferenças de vigor, pois quanto mais rápido a semente germinar, maior o vigor (Marcos Filho, 2005). Esta diferenciação também foi observada no presente trabalho (Tabela 1), mostrando que o lote 1 apresentou baixa velocidade na emergência de plântulas, refletindo uma proporção elevada de sementes que se mostram mais sensíveis às variações de temperatura e umidade relativa do ambiente a qual estão submetidas.

O teste de primeira contagem de emergência também é considerado um teste de vigor, por estar relacionado com o processo de deterioração. Durante esse processo, a velocidade de emergência decai antes da porcentagem de emergência (Malone et al., 2008). Assim, os lotes 2, 3 e 4 (Tabela 1), que emergiram mais rapidamente e apresentaram valores mais elevados de emergência na primeira contagem, podem ser considerados mais vigorosos que aqueles de emergência mais lenta (Matthews, 1980; Silva \& Vieira, 2006).

No presente trabalho, os lotes mais vigorosos apresentaram sementes que originaram plântulas com maior taxa de crescimento e maior massa seca (Tabela 1). O acúmulo de massa seca é considerado um parâmetro importante para analisar o vigor de sementes, pois permite determinar a transferência de massa seca dos tecidos de reserva para o eixo embrionário, através do peso da massa seca da plântula (Krzyzanowski et al., 1999).

Os lotes que apresentaram valores mais altos no teste de frio (lotes 2, 3 e 4), podem ser considerados mais vigorosos. Os resultados do teste de frio geralmente são semelhantes àqueles observados em campo e, por ser conduzido em condições estressantes, tem maior sensibilidade em prognosticar o desempenho das sementes (Cícero \& Vieira, 1994).

A alta condutividade elétrica do lote 4 ocorreu devido aos lixiviados em soluções nas quais as sementes foram imersas, resultado da liberação de mais exsudatos para o exterior da célula, consequentemente, apresentando maior deterioração das membranas celulares das sementes e menor vigor (Marcos Filho, 2005).

Quando se utiliza uma maior quantidade de sementes ocorre melhor estratificação dos lotes em diferentes níveis de vigor (Araújo et al., 2011). Para moringa, a combinação de 50 sementes proporcionou uma melhor separação dos lotes em diferentes níveis de vigor.

Em relação ao período de imersão das sementes de moringa, pode-se verificar o aumento na liberação de eletrólitos liberados pelas sementes com o transcorrer do tempo de imersão. O aumento nos valores de condutividade elétrica com o aumento do tempo de embebição já foi reportado anteriormente. Marques et al. (2002), trabalhando com sementes de Dalbergia nigra Fr. Allem. (jacarandá-da-bahia), observaram aumento nos valores de condutividade elétrica com o aumento do tempo de embebição, independente da temperatura e da qualidade do lote de sementes. Em contrapartida, estes resultados não foram verificados nos trabalhos de Ataide et al. (2012) e Pereira \& Martins Filho (2012) que observaram variação irregular entre os períodos de avaliação. A resposta diferenciada indica a necessidade de condução desse teste para cada espécie de interesse.

Os valores da condutividade elétrica diminuíram à medida que houve aumento do volume de água utilizado. Resultados semelhantes foram observados por Oliveira et al. (2012) em sementes de girassol.

De forma geral, para as várias combinações, volume de água/quantidade de sementes/período de embebição, houve aumento da quantidade de eletrólitos lixiviados no intervalo de zero a $24 \mathrm{~h}$. Essa constatação também foi verificada para sementes de Eruca sativa L. (rúcula) (Torres \& Pereira, 2010), Curcubita moschata Duch. 
(abóbora) (Vieira \& Dutra, 2006) e de Capsicum baccatum L. (pimenta) (Vidigal et al., 2008). No entanto, a classificação dos lotes tornou-se mais evidente com a utilização de 50 sementes e volume de água destilada de $75 \mathrm{~mL}$ nos períodos de 4, 8, 12, 16 e $24 \mathrm{~h}$ e, 50 sementes em $125 \mathrm{~mL}$ por $4 \mathrm{~h}$, possibilitando, dessa forma, separar os quatro lotes avaliados em quatro níveis de vigor.

A separação mais nítida dos lotes em diferentes níveis de vigor também foi possível quando utilizada maior quantidade de sementes. Isso pode ser explicado pelo fato de que a maior quantidade de sementes gera maior liberação de eletrólitos (Silva et al., 2014).

É importante ressaltar que o período reduzido de embebição das sementes de moringa para a realização do teste de condutividade elétrica foi satisfatório, possibilitando a obtenção de resultados mais rápidos. A redução do tempo de embebição para a avaliação da condutividade elétrica em diferentes espécies é tema de várias pesquisas (Dutra \& Vieira, 2006; Menezes et al., 2007; Vidigal et al., 2008; Alves \& Sá, 2009), pois a indústria de sementes exige, cada vez mais, procedimentos eficientes e rápidos para caracterização de lotes em relação à sua qualidade.

\section{Conclusão}

O teste de condutividade elétrica adaptado para Moringa oleífera mostrou-se eficiente em ranquear os lotes de sementes em diferentes níveis de vigor, podendo ser usado com eficiência na avaliação da sua qualidade fisiológica.

\section{Referências}

Alves, C. Z. \& Sá, M. E. Teste de condutividade elétrica na avaliação do vigor de sementes de rúcula. Revista Brasileira de Sementes, v. 31, n. 1, p. 203-215, 2009. DOI: 10.1590/S010131222009000100023.

Araújo, R. F. et al. Teste de condutividade elétrica para sementes de feijão-mungo-verde. Revista Brasileira de Sementes, v. 33, n. 1, p. 123-130, 2011. DOI: 10.1590/S0101-31222011000100014.

Ataide G. M. F. et al. Adequação da metodologia do teste de condutividade elétrica para sementes de Pterogyne nitens Tull. Revista Brasileira de Ciências Agrárias, v. 7, n. 4, p. 635-640, 2012. DOI: 10.5039/agraria.v7i4a1688.

Brasil. Ministério da Agricultura, Pecuária e Abastecimento. Secretaria de Defesa Agropecuária. Regras para análise de sementes. Brasília, DF, 2009. 395 p.
Cícero, S. M. \& Vieira, R. D. Teste de frio. In: Vieira, R. D. \& Carvalho, N. M. (Ed.). Testes de vigor em sementes. Jaboticabal: FUNEP, 1994. p. 151-164.

Dutra, A. S. \& Vieira, R. D. Teste de condutividade elétrica para a avaliação do vigor de sementes de abobrinha. Revista Brasileira de Sementes, v. 28, n. 2, p. 117-122, 2006. DOI: 10.1590/S010131222006000200015 .

Ferreira, P. M. P. et al. Moringa oleifera: bioactive compounds and nutritional potential. Revista de Nutrição, v. 21, n. 4, p. 431-437, 2008. DOI: $10.1590 / \mathrm{S} 1415-52732008000400007$.

Karadi, R. V. et al. Effect of Moringa oleifera Lam. rootwood on ethylene glycol induced urolithiasis in rats. Journal of Ethnopharmacology, v. 105, n. 1. p. 306-311, 2006. DOI: 10.1016/j. jep.2005.11.004.

Krzyzanowski, F. C. et al. Relato dos testes de vigor disponíveis para as grandes culturas. Informativo Abrates, v. 1, n. 2, p. 15-50, 1991.

Krzyzanowski, F. C. et al. Vigor de sementes: conceitos e testes. Londrina: Abrates, 1999. 218 p.

Maguire, J. D. Speed of germination-aid in evaluation for seedling emergence and vigour. Crop Science, v. 2, n. 2, p. 176-177, 1962.

Malone, P. F. V. A. et al. Potencial fisiológico de sementes de mogango e desempenho das plantas no campo. Revista Brasileira de Sementes, v. 30, n. 2, p. 123-120, 2008. DOI: 10.1590/S010131222008000200015 .

Marcos Filho, J. Fisiologia de sementes de plantas cultivadas. Piracicaba: Fealq, 2005. 496 p.

Marcos Filho, J. \& Novembre, A. D. L. C. Avaliação do potencial fisiológico de sementes de hortaliças. In: Nascimento, W. M. (Ed). Tecnologia de sementes de hortaliças. Brasília, DF: Embrapa Hortaliças, 2009. p. 185-243

Marques, M. A. et al. Adequação do teste de condutividade elétrica para determinar a qualidade fisiológica de sementes de jacarandá-dabahia (Dalbergia nigra Vell.) Fr. All. ex. Benth). Revista Brasileira de Sementes, v. 24, n. 1, p. 271-278, 2002. DOI: 10.1590/S010131222002000100038 .

Matthews, S. Controlled deterioration: a new vigour test for crop seeds. In: Habblethwait, P. D. Seed production. London: Butterworths, 1980. p. 647-660.

Menezes, N. L. et al. Teste de condutividade elétrica em sementes de aveia preta. Revista Brasileira de Sementes, v. 29, n. 2, p. 138-142, 2007. DOI: 10.1590/S0101-31222007000200019.

Nakagawa, J. Testes de vigor baseados no desempenho das plântulas. In: Krzyzanoski, F. C. et al. (Ed.). Vigor de sementes: conceitos e testes. Londrina: Abrates, 1999. p. 1-24.

Oliveira, F. N. et al. Qualidade fisiológica de sementes de girassol avaliadas por condutividade elétrica. Pesquisa Agropecuária Tropical, v. 42, n. 3, p. 279-287, 2012.

Pereira, M. D. \& Martins Filho, S. Adequação da metodologia do teste de condutividade elétrica para sementes de cubiu (Solanum sessiflorum DUNAL). Revista Agrarian, v. 5, n. 16, p. 93-98, 2012. 
Ribeiro, D. M. et al. Teste de condutividade elétrica para avaliar o vigor de sementes em milho-pipoca (Zea mays L.). Revista Ceres, v. 56, n. 6 , p. $772-776,2009$.

Rodrigues, M. B. C. et al. Pré-hidratação em sementes de soja e eficiência do teste de condutividade elétrica. Revista Brasileira de Sementes, v. 28, n. 2, p. 168-181, 2006.

Silva, J. B. \& Vieira, R. D. Avaliação do potencial fisiológico de sementes de beterraba. Revista Brasileira de Sementes, v. 28, n. 3, p. 128-134, 2006.

Silva, J. E. N. et al. Teste de condutividade elétrica para sementes de feijão manteiguinha. Ensaios e Ciências: Ciências Biológicas, Agrárias e da Saúde, v. 17, n. 6, p. 37-46, 2014.

Sponchiado, J. C. Teste de condutividade elétrica para determinação do potencial fisiológico de sementes de aveia branca. Ciências Agrárias, v. 35, n. 4, p. 2405-2414, 2014. DOI: 10.5433/1679-0359.2014v35n4Sup11p240.

Tokuhisa, D. et al. Teste de condutividade elétrica para avaliação da qualidade fisiológica de sementes de mamão (Carica papaya L.). Revista Brasileira de Sementes, v. 31, n. 2, p. 137-145, 2009. DOI: $10.1590 / \mathrm{S} 0101-31222009000200016$.
Torres, S. B. \& Pereira, R. A. Condutividade elétrica em sementes de rúcula. Revista Brasileira de Sementes, v. 32, n. 4, p. 58-70, 2010. DOI: $10.1590 / \mathrm{S} 0101-31222010000400007$.

Valadares, J. \& Paula, R. C. Temperaturas para germinação de sementes de Poecilanthe parviflora Benthan (Fabaceae-Faboideae). Revista Brasileira Sementes, v. 30 n. 2, p. 164-170, 2008. DOI: 10.1590/S0101-31222008000200020

Vidigal, D. S. et al. Teste de condutividade elétrica para semente de pimenta. Revista Brasileira de Sementes, v. 30, n. 1, p. 168-174. 2008. DOI: 10.1590/S0101-31222008000100021.

Vieira, R. D. \& Dutra, A. D. Condutividade elétrica em sementes de abóbora, híbrido Bárbara. Horticultura Brasileira, v. 24, n. 3, p. 305-308, 2006. DOI: 10.1590/S0102-05362006000300007.

Vieira, R. D. et al. Condutividade elétrica e teor de água inicial das sementes de soja. Pesquisa Agropecuária Brasileira, v. 37, n. 9, p. 1333-1338, 2002. DOI: 10.1590/S0100-204X2002000900018. 
